
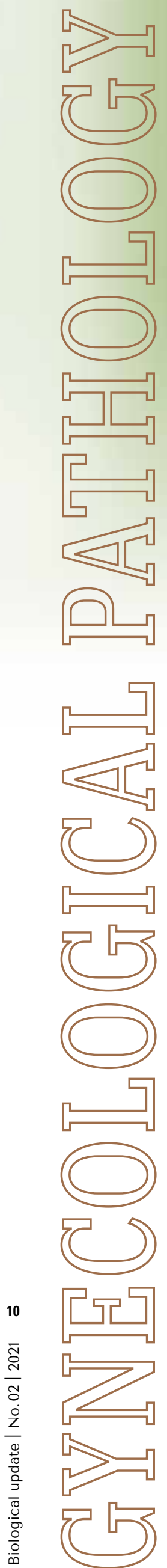

\title{
Features of the
} morphological structure of the placenta in pregnant women with preeclampsia

\author{
DOI 10.52739/bio-up.2.2021.10-15
}

Volodymyr I. Chermak', Ihor I. Chermak², Chukwuanyinonso O. Ikeotuonye ${ }^{3}$, Anatolii Ya. Senchuk'

\section{SUMMARY}

o study morphological features of the placenta in women in labor, pregnancy which complicated by preeclampsia we studied 26 placentae, of which 17 were from pregnant women with mild to moderate preeclampsia (basic group) and 9 from healthy women (control group). Histologically the study was conducted with methodically recommendations of T.D. Zadorozhnaya et al. for light microscopy.

During the study of morphological features of the placenta in women in labor with preeclampsia were installed morphological changes in the placenta, which reflect a tendency to develop compensatory-adaptive reactions following their stress and exhaustion,

1 Department of Obstetrics and Gynecology, Private Higher Education Establishment Kyiv Medical University, Kyiv, Ukraine

2 Department of Obstetrics and Gynecology and Neonatology of the Institute of Postgraduate Education, Bogomolets National Medical University, Kyiv, Ukraine

3 Department of Pathological Anatomy, Histology and Forensic Medicine, Private Higher Education Establishment Kyiv Medical University, Kyiv, Ukraine 
which is a sign of placental insufficiency. Received data allow claiming a high frequency of development placental insufficiency in pregnant women with risk factors such as chronic salpingo-oophoritis, anemia, late preeclampsia, and inflammation diseases kidney.

Keywords: pregnancy, preeclampsia, placental insufficiency, pathomorphology of placenta.

Placental insufficiency is one of the most important problems of modern obstetrics and perinatology and is one of the leading causes of perinatal morbidity and mortality (Makatsariya, Chervenaka, \& Bitsadze, 2015). Placental insufficiency (PI) is a fairly common pathology (Yamamoto et al., 2018). It manifests itself in 3-4\% healthy women with uncomplicated pregnancy (Guise, 2007), and in this case, diverse pathology frequency fluctuates from 24 to $46 \%$ (Morgan, 2014). PI is one of the most important problems of modern obstetrics and perinatology and occupies one of the first places among the causes of perinatal morbidity and mortality Boeldt, D. S., \& Bird, 2017). According to modern results research (Bucklin, Gambling, \& Wlody, 2008), 20-60\% cases of perinatal mortality are directly associated with the pathology placenta (Mihu, Razvan, Malutan, \& Mihaela, (2015).

The problem of $\mathrm{PI}$ basically is violations of adaptive mechanisms and is still relevant, associated with negative impact on newborn and numerous complications during childbirth (Myatt et al., 1996), PI can lead to numerous of disorders of nervous system, and pose child at risk of somatic and infectious pathologies (Adriichuk, Gavriushov, Senchuk, \& Volodymyr, 2021).

The most common reasons for the development of PI are extragenital and obstetric pathology of the mother during pregnancy (Roland et al., 2016), which leads to violations of uterine-placental, and later fetoplacental circulation (IM, DS, MA, FX, \& RR, 2013). These circumstances may lead to the development of irreversible morphological processes and violated functions placenta (Kimura et al., 2019).

One of the most important risk factor for the development of $\mathrm{PI}$ is pregnancy complicated by preeclampsia.
Recently observed growth of scientific interest of impact factors risk to the state of the fetoplacental complex, which determines violation uterine-placental hemodynamics as a key link in the pathogenesis of PI (Chermak, Chermak, Matvian, \& Singh, 2021).

The purpose of current research was to study morphological features of the placenta in women in labor whose pregnancy was complicated by mild to moderate preeclampsia.

\section{MATERIALS AND METHODS}

We researched 26 placentae, of which 17 were from pregnant women with mild to moderate preeclampsia (main group), and 9 from healthy women (control group). To assess the condition of the placenta, a histological examination was performed according to the methodological recommendations of T. D. Zadorozhnaya et al. (Saha, Biswas, Mitra, Adhikari, \& Saha, 2014). For light microscopy. Placental tissues were stained with $\mathrm{H} \& \mathrm{E}$ and by the method of Van-Gizon (picrotoxin). Ultrastructural analysis was conducted under a light microscope from built-in digital camera "OLYMPUS And-WITH MAD3 (Japan) with standard magnifications: $x 40, x 100, x 200, x 400$.

Research results and their discussion. We paid attention to the features of placental abruption and excretion manure in patients' main and control groups. Defects manure was observed in $47.1 \%$ cases (8 placentae) patients with preeclampsia against $11.1 \%$ ( 1 case) placenta healthy patients.

Macroscopic picture: maternal surface of placenta in main group where smooth, dark-red color, with pronounced lobed. Maternal surface differed uneven full-blooded (70.6\%). IN 7 (41.2\%) cases throughout surface visualized foci microinfarctions $(0.4-0.6 \mathrm{~cm}$ in diameter). During the examination of the placenta, healthy pregnant women had similar foci found in $11.1 \%$ of cases (1 placenta). Almost all placentas of the main group (88.2\%) observed with considerable maternal calcification surface in the form of fine whitish inclusions (in the control group this indicator made up $22.2 \%$ ).

Fetal surface of the placenta of patients both groups did not differ: smooth, brilliant, gray 
color. During the collection of samples for histological study there were found that placental tissue is brown-red color, spongy structures. Attachment umbilical cord to the placenta were found in $94.1 \%$ (16 cases of main groups) central. In $12.8 \%$ we detected long (70 and $75 \mathrm{~cm}$ ) and in $3(17.6 \%)$ cases short $(37$, $39,40 \mathrm{~cm}$ ) umbilical cord in patients with preeclampsia. The surface of the umbilical cord was brilliant, smooth. Hematomas and nodules on the umbilical cord were not detected. At incision umbilical cord in all cases two arteries and one vein were detected. Other differences in the above indicators between groups were not found.

Placenta parturient with late gestosis had small reliably reduced mass compared with healthy placentas women, which was $430.35+13.67 \mathrm{~d}$ $(p<0.05)$, what reliably more $(p<0.05)$, than in the control group $-554.6 \pm 18.1 \mathrm{~d}$.

Feto-placental coefficient (FPC) varied within $0.14-0.22$. We have established an significant increase in FPC in the main group $-0.21 \pm$ 0.02 compared with the control group -0.18 \pm 0.02 , which explained higher birth rate children with low weight body and in asphyxia for enlargement secondary placental mass in women with preeclampsia.

The study revealed significant structural changes in the histological structure of the placenta in patients, whose pregnancy was complicated by preeclampsia. First of all, we observe a significant increase in the number of small villi (the cross-section of which has a diameter of 11 to $90 \mu \mathrm{m})$. Significantly increased packing density of the cytotrophoblast, stroma and vascular villi, intervillous space, as well as the volume of non-functional tissue - fibrinoid, hemorrhages, calcifications, and basal necrosis, sometimes - the chorionic plate, angiomatosis of intermediate villi with stasis of erythrocytes. In addition, detected expression refinement and desquamation cytotrophoblast, magnification percent intermediate immature chorionic villi. The walls of the villi vessels are located directly near the cytotrophoblast, which indicates the formation of syncytio-capillary membranes.

Histological examination of the placenta in women in labor, pregnancy whose complicated late gestosis, allowed to allocate the dominant pathological picture immaturity villous tree - predominance intermediate and chaotic $c_{11}$ small hairs with multiple sclerosis on the background reduction terminal villi.

Morphological equivalent local tissue hypoxia became significant magnification syncytial nodules, the majority of them corresponded to the so-called involutive forms, which indicates the chronic nature of the local hypoxia.

Also in micro preparations placenta with late gestosis reliably more often $(p<0.05)$ met villi groups, what walled up fibrinoid, - pseudoinfarctions.

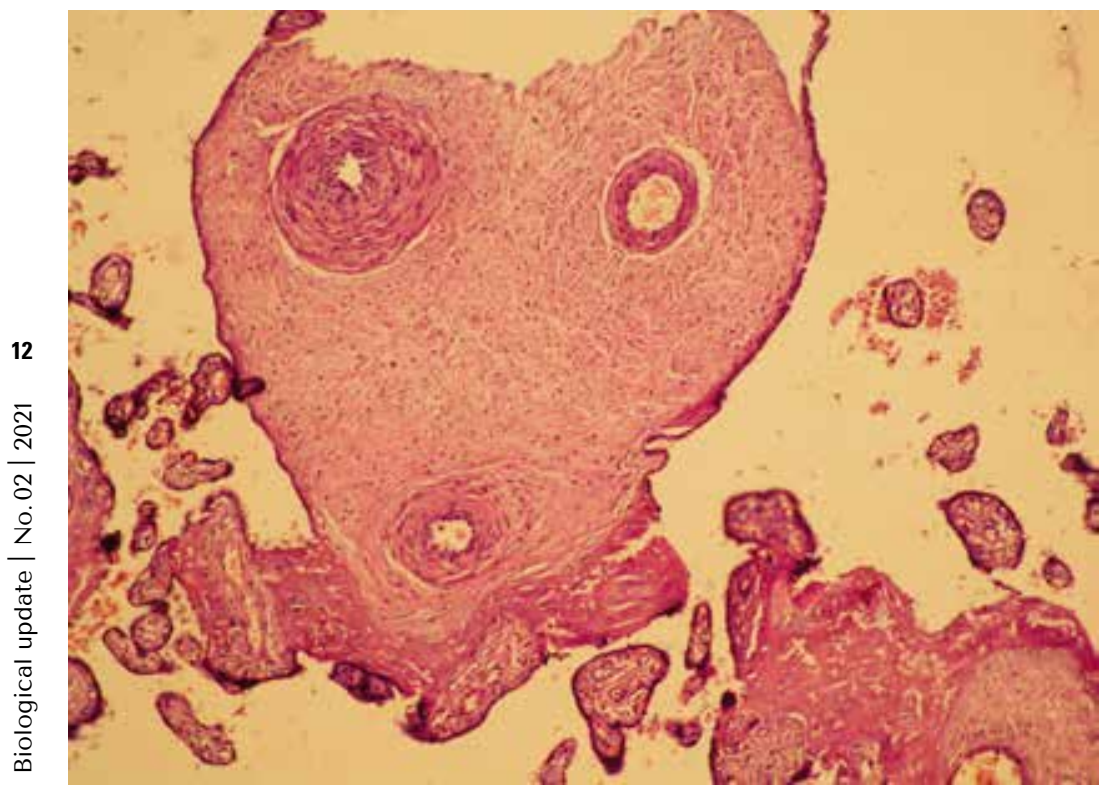

Figure 1. Placenta of patient with moderate preeclampsia during 36-37 weeks of pregnancy. Secondary villi with hyperplasia of endothelial cells and hyperplasia of smooth muscle cells network and the tendency to obliteration. Areas of distally located villi, walled up fibrinoid. H\&E staining, magnification x 200 . 
Figure 2. Placenta of patient from main group with mild preeclampsia in time 39 weeks of pregnancy. Pseudoinfarction with full loss of epithelium in the villi, single cells cytotrophoblast with pronounced dystrophy. H\&E staining, magnification $\times 200$.
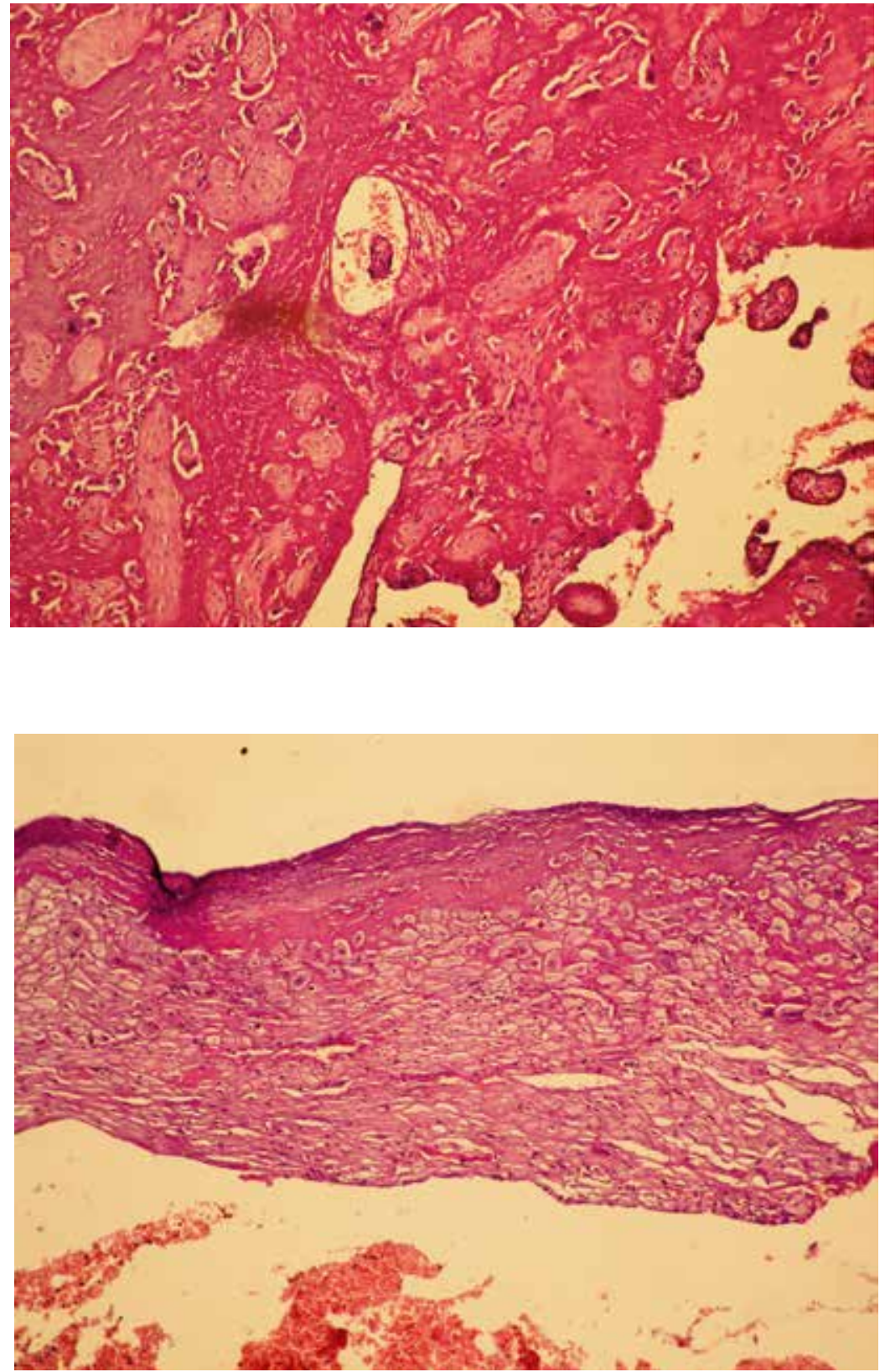

Figure 3. Placenta of patient from main group with mild preeclampsia in time 37-38 weeks of pregnancy. Expressed fibrinoid necrosis of the basal plate of the placenta, disorganization of decidual cells and necrosis foci. H\&E staining, magnification $\times 200$

Figure 4. Placenta of patient from main group with mild preeclampsia in time 38 weeks of pregnancy. Embryonic villi with proliferation of cytotrophoblast. $H \&$ E staining, magnification $\times 200$.

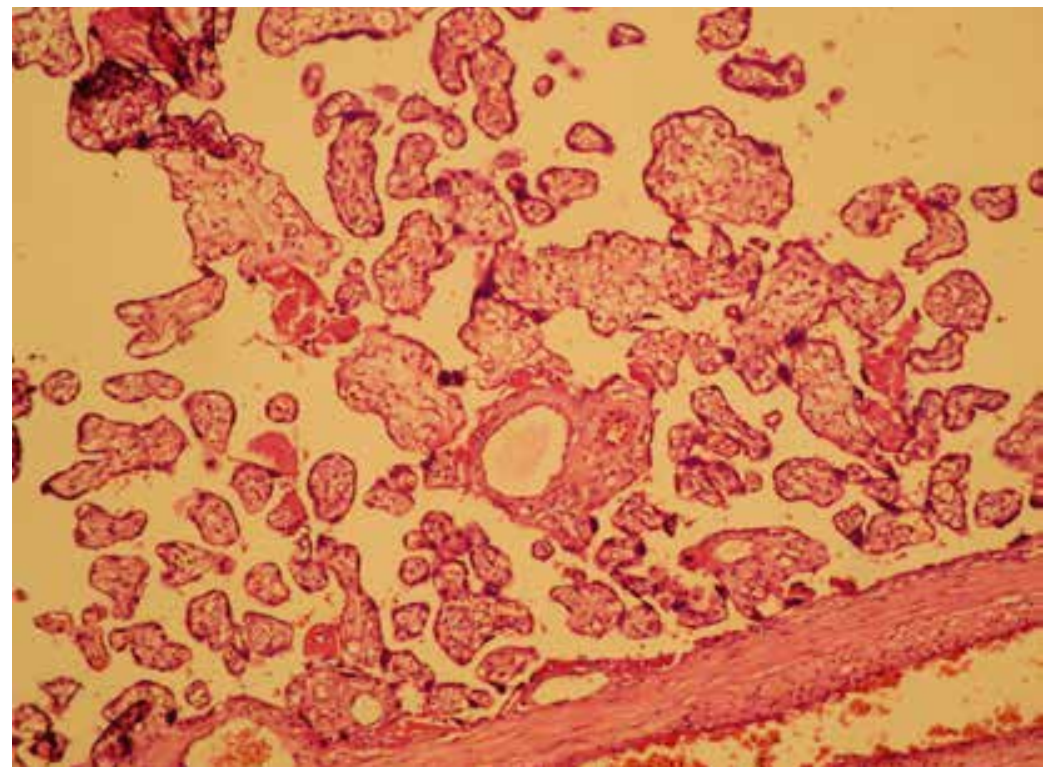


Characteristic histological sign of placenta with preeclampsia was the presence of significant quantity $(46.0 \%)$ of small foci of infarcts.

Specified changes causally due to obliterative angiopathy in the proximal departments hairy tree.

Compensatory-adaptive processes in the placenta from main group had manifestations in the form of formation large quantity of syncytial nodules and hypervascularized terminal villi.

Accordingly, detected changes in the placenta of women with preeclampsia indicate the presence of compensated chronic placental dysfunction.

Along with this, in the placenta patients with preeclampsia we have found adaptive reactions also, thanks to which was provided fetal viability. This was reflected in the availability of terminal villi, which experienced hypertrophy, with branched grid full-blooded capillaries in combination with the expressed decrease intervillous space. Compensatory changes terminal villi had uneven in nature and were more expressed in the central and paracentral departments. In some cases, data changes was found in intermediate villi, with number of terminal villi was much reduced, which indicates premature maturation placenta.

\section{CONCLUSIONS}

Analysis of the placenta of women with late gestosis demonstrates the combined nature of the lesion of placental tissue and indicates the presence of compensated, and in some cases decompensated chronic placental insufficiency. Taking into account the identified signs of placenta-uterine dysfunction (a variant of violation of villi maturation and variant of hypovascularized chorionic villi), we see a causal relationship between morphological changes in the placenta with the development of fetal distress.

Detected pathological changes in the placenta undoubtedly contribute to development of complications during pregnancy, childbirth, and understatement compensatory-adaptive ability of newborns.
Our research gives grounds to claim the substantial difference between indicators in patients from main and control groups, which show a decrease compensatory capabilities of the fetus in pregnant women with preeclampsia and indicates appropriateness for more profound observation of the condition of the fetus in this category of pregnant women.

\section{REFERENCES}

Makatsariya, A. D., Chervenaka, F. A., \& Bitsadze, V. O. (2015). Beremennost'vysokogo riska. M.: OOO «MIA.

Yamamoto, J. M., Hughes, D. J., Evans, M. L., Karunakaran, V., Clark, J. D., Morrish, N. J., ... \& Murphy, H. R. (2018). Community-based pre-pregnancy care programme improves pregnancy preparation in women with pregestational diabetes. Diabetologia, 61(7), 15281537.

Morgan, T. K. (2014). Placental insufficiency is a leading cause of preterm labor. NeoReviews, 15(12), e518-e525.

Guise, J. M. (2007). Anticipating and responding to obstetric emergencies. Best Practice \& Research Clinical Obstetrics \& Gynaecology, 21(4), 625-638.

Bucklin, B. A., Gambling, D. R., \& Wlody, D. (Eds.). (2008). A practical approach to obstetric anesthesia. Lippincott Williams \& Wilkins.

Boeldt, D. S., \& Bird, I. M. (2017). Vascular adaptation in pregnancy and endothelial dysfunction in preeclampsia. The Journal of endocrinology, 232(1), R27.

Mihu, D., Razvan, C., Malutan, A., \& Mihaela, C. (2015). Evaluation of maternal systemic inflammatory response in preeclampsia. Taiwanese Journal of Obstetrics and Gynecology, $54(2), 160-166$.

Myatt, L., Rosenfield, R. B., Eis, A. L., Brockman, D. E., Greer, I., \& Lyall, F. (1996). Nitrotyrosine residues in placenta: evidence of peroxynitrite formation and action. Hypertension, 28(3), 488-493. 
Andriichuk, T., Gavriushov, D., Senchuk, A., \& Volodymyr, C. (2021). Pathomorphological and Ultrastructural Placental Changes in Women with a High Risk of Placental Insufficiency. Annals of the Romanian Society for Cell Biology, 84-89.

Roland, C. S., Hu, J., Ren, C. E., Chen, H., Li, J., Varvoutis, M. S., ... \& Jiang, S. W. (2016). Morphological changes of placental syncytium and their implications for the pathogenesis of preeclampsia. Cellular and molecular life sciences, 73(2), 365-376.

IM, B., DS, B., MA, G., FX, Y., \& RR, M. (2013). Pregnancy, programming and preeclampsia: gap junctions at the nexus of pregnancy-induced adaptation of endothelial function and endothelial adaptive failure in PE. Current vascular pharmacology, 11(5), 712-729.
Kimura, F., Takebayashi, A., Ishida, M., Nakamura, A., Kitazawa, J., Morimune, A., ... \& Murakami, T. (2019). Chronic endometritis and its effect on reproduction. Journal of Obstetrics and Gynaecology Research, 45(5), 951960.

Chermak, I. I., Chermak, V. I., Matvian, M. A., \& Singh, M. (2021). The role of chronic endometritis in development of infertility in terms of etiological factors. Biomedical update, (1), $22-27$.

Saha, S., Biswas, S., Mitra, D., Adhikari, A., \& Saha, C. (2014). Histologic and morphometric study of human placenta in gestational diabetes mellitus. Histologic and morphometric study of human placenta in gestational diabetes mellitus, 1-9.

\section{Corresponding author:}

Anatolii Ya. Senchuk, 0509453723@ukr.net

Manuscript was recieved on 10 September; accepted for publication on 2 November. 